\title{
Classification of Hand Movements based on Discrete Wavelet Transform and Enhanced Feature Extraction
}

\author{
Jingwei Too ${ }^{1}$, Abdul Rahim Abdullah ${ }^{2}$, Norhashimah Mohd Saad ${ }^{3}$ \\ Fakulti Kejuruteraan Elektrik, Universiti Teknikal Malaysia Melaka, Melaka, Malaysia ${ }^{1,2}$ \\ Fakulti Kejuruteraan Elektronik dan Kejuruteraan Komputer, Universiti Teknikal Malaysia Melaka, Melaka, Malaysia ${ }^{3}$
}

\begin{abstract}
Extraction of potential electromyography (EMG) features has become one of the important roles in EMG pattern recognition. In this paper, two EMG features, namely, enhanced wavelength (EWL) and enhanced mean absolute value (EMAV) are proposed. The EWL and EMAV are the modified version of wavelength (WL) and mean absolute value (MAV), which aims to enhance the prediction accuracy for the classification of hand movements. Initially, the proposed features are extracted from the EMG signals via discrete wavelet transform (DWT). The extracted features are then fed into the machine learning algorithm for classification process. Four popular machine learning algorithms include $\boldsymbol{k}$-nearest neighbor (KNN), linear discriminate analysis (LDA), Naïve Bayes (NB) and support vector machine (SVM) are used in evaluation. To examine the effectiveness of EWL and EMAV, several conventional EMG features are used in performance comparison. In addition, the efficacy of EWL and EMAV when combine with other features are also investigated. Based on the results obtained, the combination of EWL and EMAV with other features can improve the classification performance. Thus, EWL and EMAV can be considered as valuable tools for rehabilitation and clinical applications.
\end{abstract}

Keywords-Electromyography; feature extraction; discrete wavelet transform; classification; pattern recognition

\section{INTRODUCTION}

As a biomedical signal, electromyography (EMG) signal is playing an important role in developing the human machine interaction devices. Naturally, EMG signal recorded from the muscle contraction contains rich muscle information, which is beneficial in describing the muscle behavior and condition, as well as the hand movement [1], [2]. In recent days, the myoelectric control has been received much attentions from the biomedical researchers. The correlation between amplitude and motion grants the EMG signal to become one of the most powerful sources in controlling the prosthesis [3].

Thanks to current technology, the usage of pattern recognition based myoelectric control has become viable. Needless to say, the type of classifier does not significantly affect the classification performance, while the quality of extracted features has shown a great impact in EMG signals classification [4]. Without loss of generality, feature extraction is a technique to extract the valuable information from the signal itself, which should contain much information as possible [4], [5]. Feature extraction can be categorized into time domain (TD), frequency domain (FD) and time-frequency domain (TFD). Among the EMG features, TD features are the most commonly used. In a past study, Hudgins et al. [6] introduced five EMG features for pattern recognition. The authors indicated that proposed features are good in discriminating the EMG patterns. Later, Khushaba et al. [7] developed a subset of features based on time-dependent spectral moments to classify the multiple hand movements at different limb positions. Moreover, Samuel et al. [8] proposed three new EMG features for arm movements classification. The author showed that proposed features outperformed other conventional features in EMG pattern recognition. However, TD features assume the EMG data from stationary signals and there is no frequency information provided [9]. On one hand, FD features only contains spectral information, in which the time information is limited. For these reasons, the TFD features are utilized in this work.

In the past studies, time-frequency methods such as short time Fourier transform (STFT) and wavelet transform (WT) are widely used in EMG signal processing [10]-[12]. However, STFT cannot provide satisfactory performance due to its fixed window size [13], [14]. From the previous works, it is reported that spectrogram or short time Fourier transform (STFT) was not very effective in EMG pattern recognition system [10], [13]. As compared to STFT, wavelet transform (WT) provides changeable time and frequency resolution, which is more formidable for extracting the high quality signal information [14]. In this regard, we focus on WT in current work.

Generally, WT decomposes the signal into detail and approximation coefficients at different sub-bands, in which the time information at different frequency ranges can be obtained [14]. Previous works indicated that the features extracted based on discrete wavelet transform (DWT) were showing good discriminate power in describing the target concept. Phinyomark et al. [15] extracted the mean absolute value (MAV) feature via DWT transformation for patterns classification. Doulah et al. [16] applied the DWT and root mean square (RMS) feature for neuromuscular diseases classification. Moreover, Xing et al. [9] proposed the wavelet packet node energy as the features for EMG pattern recognition. Previous findings showed that the feature extraction based on WT transformation was more capable in achieving a high classification performance.

Among the existing EMG features, wavelength (WL) and mean absolute value (MAV) are the most frequently used. This is mainly due to their efficiency and simplicity in EMG pattern recognition [1], [15]. Based on WL and MAV features, we propose the enhanced wavelength (EWL) and enhanced mean absolute value (EMAV) features in this paper. The proposed features are tested by using the EMG data acquired from the 
publicly access EMG database. Four popular machine learning algorithms include $k$-nearest neighbor (KNN), Naïve Bayes (NB), linear discriminate analysis (LDA) and support vector machine (SVM) are used in evaluation. In addition, several conventional EMG features are used in performance comparison. Moreover, the effectiveness of EWL and EMAV when combine with other features are also investigated. The experimental results show that EWL and EMAV can be valuable tools for rehabilitation and clinical applications.

The organization of paper as follows: Section II describes the proposed EMG pattern recognition system. Meanwhile, the proposed feature extraction methods are also presented. Section III discusses the experimental results. At last, the conclusion is pointed in Section IV.

\section{MATERIAL AND METHOD}

Fig. 1 illustrates the flow diagram of proposed EMG pattern recognition system. Firstly, the EMG signals are acquired from the publicly access EMG database. Afterward, DWT is applied to decompose the signals into multiresolution wavelet coefficients. Next, the features are extracted from each coefficient to form an EMG feature set. The feature set that consists of several features are then fed into the classifiers (machine learning algorithms) for the classification of six hand movement tasks.

\section{A. EMG Data}

The EMG data is acquired from the sEMG for Basic Hand Movements Data Set via UCI Machine Learning Repository [17]. Note that only the first database is utilized in this work. This dataset consists of the EMG signals of six different hand movement tasks recorded from five healthy subjects (two males and three females). In the experiment, two channels were used in the process of recording. The subject was instructed to perform each hand movement for 6 seconds. Additionally, each movement was repeated for 30 times, and the EMG signals were sampled at $500 \mathrm{~Hz}$ [18]. The hand movement tasks are listed in Table I.

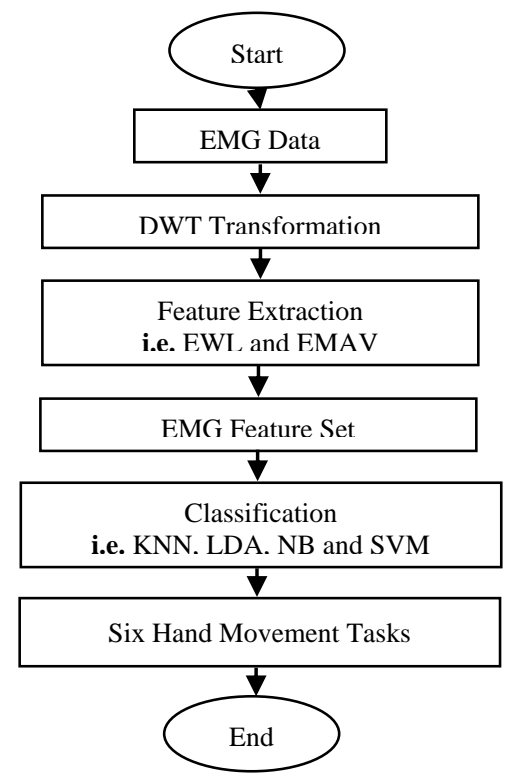

Fig. 1. Flow Diagram of Proposed EMG Pattern Recognition System.
TABLE I. LISTED HAND MOVEMENTS

\begin{tabular}{|l|l|}
\hline No & Hand movement task \\
\hline 1 & Spherical \\
\hline 2 & Tip \\
\hline 3 & Palmar \\
\hline 4 & Lateral \\
\hline 5 & Cylindrical \\
\hline 6 & Hook \\
\hline
\end{tabular}

\section{B. Discrete Wavelet Transform}

Basically, EMG signal is presented in time domain (TD). However, in TD, the frequency and spectral information are limited. Thus, the time-frequency method is applied in this research. Due to the efficiency and reliable of discrete wavelet transform (DWT) in biomedical signal processing, the DWT has become our major focus in this work. Briefly, DWT decomposes the EMG signals into multiresolution wavelet coefficients, which exhibits the signal in both time and frequency representations. By this way, the extracted features contain the time information at different frequency sub-bands [19]. This in turn will improve the time-frequency information, thus leading to high prediction accuracy.

The wavelet decomposition involves two digital filters, which are low-pass and high-pass filters. Mathematically, the first decomposition level of DWT can be expressed as:

$$
\begin{aligned}
& A[k]=\sum_{n} x[n] \cdot g[2 k-n] \\
& D[k]=\sum_{n} x[n] \cdot h[2 k-n]
\end{aligned}
$$

where $x[n]$ represents the signal, $D[k]$ is the detail and $A[k]$ is the approximation. Note that detail and approximation are the outputs of high pass and low pass filters, respectively. The decomposition process is repeated until the desired level is satisfied [11], [20]. According to the findings in [21], the DWT with Biorthogonal 3.3 at fourth decomposition level is utilized in this paper. An illustration of wavelet decomposition of DWT at fourth decomposition level is shown in Fig. 2.

\section{Conventional Feature Extraction}

Feature extraction is a process that reforms the raw EMG data into a reduced expression set of features. A quality feature shall comprise of meaningful information that can best describe the target concept in the classification process [22]. In this study, 14 popular and commonly used EMG features are utilized. These features are selected due to their simplicity and promising performances in previous works.

Mean absolute value (MAV) is one of the most popular EMG features, and it is defined as the average of the summation of absolute value of signal [6], [23]. MAV can be expressed as:

$$
\text { MAV }=\frac{1}{L} \sum_{i=1}^{L}\left|x_{i}\right|
$$

where $x$ is the wavelet coefficient and $L$ is the length of coefficient. 

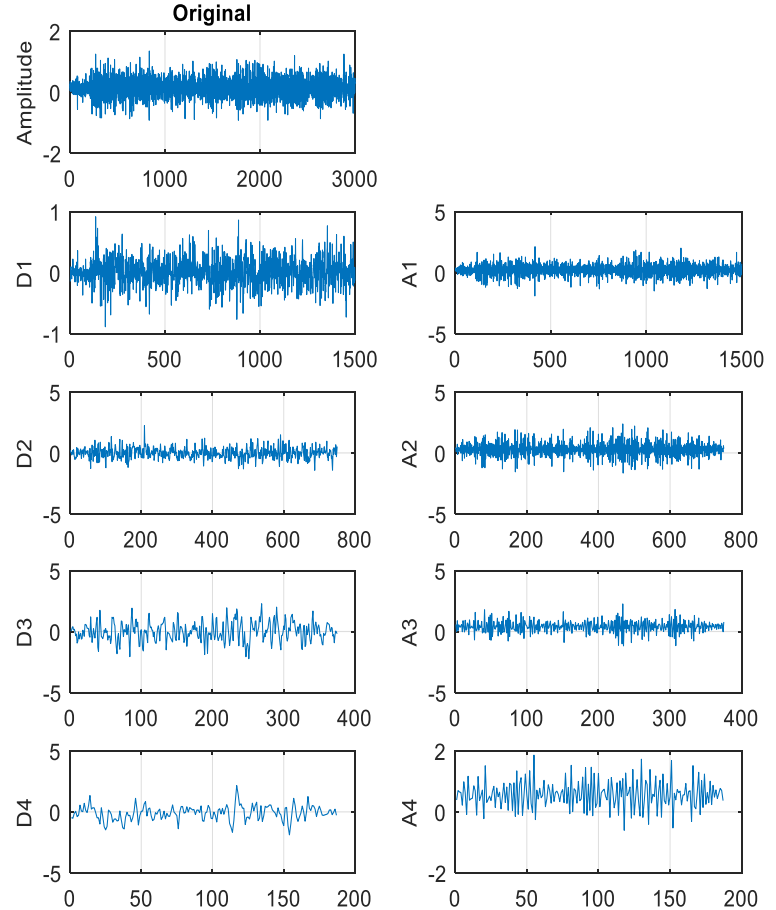

Fig. 2. Wavelet Decomposition of DWT at Fourth Decomposition Level.

Wavelength (WL) is another popular EMG feature, and it can be calculated by simplifying the cumulative length of waveform summation [6], [24]. The WL can be defined as:

$\mathrm{WL}=\sum_{i=2}^{L}\left|x_{i}-x_{i-1}\right|$

where $x$ is the wavelet coefficient and $L$ is the length of coefficient.

Zero crossing $(\mathrm{ZC})$ is an EMG feature that measures the frequency information [6], [23]. Mathematically, ZC can be expressed as:

$\mathrm{ZC}=\sum_{i=1}^{L-1} f\left(x_{i}\right)$

$f\left(x_{i}\right)=\left\{\begin{array}{l}1, \text { if }\left\{\left(x_{i}>0 \& x_{i+1}<0\right) \mid\left(x_{i}<0 \& x_{i+1}>0\right)\right\} \\ \quad \&\left|x_{i}-x_{i+1}\right| \geq T \\ 0, \text { otherwise }\end{array}\right.$

where $x$ is the wavelet coefficient, $T$ is the threshold value and $L$ is the length of coefficient.

Slope sign change (SSC) is a traditional EMG feature that determines the number of times in which the number of waveform changes sign [6], [23]. SSC can be calculated as:

$$
\begin{aligned}
\mathrm{SSC} & =\sum_{i=2}^{L-1} f\left(x_{i}\right) \\
f\left(x_{i}\right) & =\left\{\begin{array}{c}
1, \text { if }\left\{\left(x_{i}>x_{i-1} \& x_{i}>x_{i+1}\right) \mid\left(x_{i}<x_{i-1} \& x_{i}<x_{i+1}\right)\right\} \\
\&\left\{\left(\left|x_{i}-x_{i+1}\right| \geq T\right) \mid\left(\left|x_{i}-x_{i-1}\right| \geq T\right)\right\} \\
0, \text { otherwise }
\end{array}\right.
\end{aligned}
$$

where $x$ is the wavelet coefficient, $T$ is the threshold value and $L$ is the length of coefficient.

Average amplitude change (AAC) is another popular EMG feature, and it can be formulated as [24]:

$$
\mathrm{AAC}=\frac{1}{L} \sum_{i=1}^{L-1}\left|x_{i+1}-x_{i}\right|
$$

where $x$ is the wavelet coefficient and $L$ is the length of coefficient.

Log detector (LD) is a feature that is good at estimating the exerted force, and it can be defined as [25]:

$$
\mathrm{LD}=\exp \left(\frac{1}{L} \sum_{i=1}^{L} \log \left(\left|x_{i}\right|\right)\right)
$$

where $x$ is the wavelet coefficient and $L$ is the length of coefficient.

Root mean square (RMS) is one of the popular features which is useful in describing the muscle information [26]. In mathematics, RMS can be calculated as:

$$
\mathrm{RMS}=\sqrt{\frac{1}{L} \sum_{i=1}^{L}\left(x_{i}\right)^{2}}
$$

where $x$ is the wavelet coefficient and $L$ is the length of coefficient.

Difference absolute standard deviation value (DASDV) is another frequently used EMG feature, and it can be expressed as [26]:

$$
\operatorname{DASDV}=\sqrt{\frac{\sum_{i=1}^{L-1}\left(x_{i+1}-x_{i}\right)^{2}}{L-1}}
$$

where $x$ is the wavelet coefficient and $L$ is the length of coefficient.

Myopulse percentage rate (MYOP) is defined as the mean of Myopulse output in which the absolute value of EMG signal exceeds the pre-defined threshold value [24]. MYOP can be given as follows:

$$
\begin{aligned}
& \text { MYOP }=\frac{1}{L} \sum_{i=1}^{L} f\left(x_{i}\right) \\
& f\left(x_{i}\right)=\left\{\begin{array}{l}
1, \text { if } x_{i} \geq T \\
0, \text { otherwise }
\end{array}\right.
\end{aligned}
$$

where $x$ is the wavelet coefficient, $T$ is the threshold value and $L$ is the length of coefficient.

Willison amplitude (WA) is an EMG feature that acts as an indicator of the firing of motor unit potentials, and it can be computed as [25]:

$$
\begin{aligned}
& \mathrm{WA}=\sum_{i=1}^{L-1} f\left(x_{i}\right) \\
& f\left(x_{i}\right)=\left\{\begin{array}{l}
1, \text { if }\left|x_{i}-x_{i+1}\right| \geq T \\
0, \text { otherwise }
\end{array}\right.
\end{aligned}
$$


where $x$ is the wavelet coefficient, $T$ is the threshold value and $L$ is the length of coefficient.

Simple square integral (SSI) is defined as the summation of square values of EMG signal amplitude, and it can be computed as [24]:

$\mathrm{SSI}=\sum_{i=1}^{L}\left(x_{i}\right)^{2}$

where $x$ is the wavelet coefficient and $L$ is the length of coefficient.

Variance of EMG signal (VAR) is good at measuring the signal power, and it can be expressed as [25]:

$\mathrm{VAR}=\frac{1}{L-1} \sum_{i=1}^{L}\left(x_{i}\right)^{2}$

where $x$ is the wavelet coefficient and $L$ is the length of coefficient.

Modified mean absolute value (MMAV) is an extension of MAV feature by assigning the weight window function. Mathematically, MMAV can be computed as [24]:

MMAV $=\frac{1}{L} \sum_{i=1}^{L} w_{i}\left|x_{i}\right|$

$w_{i}=\left\{\begin{aligned} 1, & \text { if } 0.25 L \leq i \leq 0.75 L \\ 0.5, & \text { otherwise }\end{aligned}\right.$

where $x$ is the wavelet coefficient and $L$ is the length of coefficient.

Modified mean absolute value 2 (MMAV2) is another extension of MAV feature by assigning the continuous weight window function, and it can be expressed as [24]:

$$
\begin{aligned}
& \text { MMAV2 }=\frac{1}{L} \sum_{i=1}^{L} w_{i}\left|x_{i}\right| \\
& w_{i}= \begin{cases}1, & \text { if } 0.25 L \leq i \leq 0.75 L \\
4 i / L, & \text { if } i<0.25 L \\
4(i-L) / L, & \text { otherwise }\end{cases}
\end{aligned}
$$

where $x$ is the wavelet coefficient and $L$ is the length of coefficient.

\section{Proposed Enhanced Feature Extraction}

In this paper, two features namely enhanced mean absolute value (EMAV) and enhanced wavelength (EWL) are proposed for EMG signals classification. In the first step, the motivation of this work is briefly explained. Fig. 3 demonstrates an example of EMG signal. As can be seen, most of the information is found within the middle region of the signal. On one hand, the signal presented at the early and final stages are less informative due to slow reaction of the subject in the experiment.

To overcome the issues above, EMAV and EWL features are proposed. The proposed features are formulated as follows:

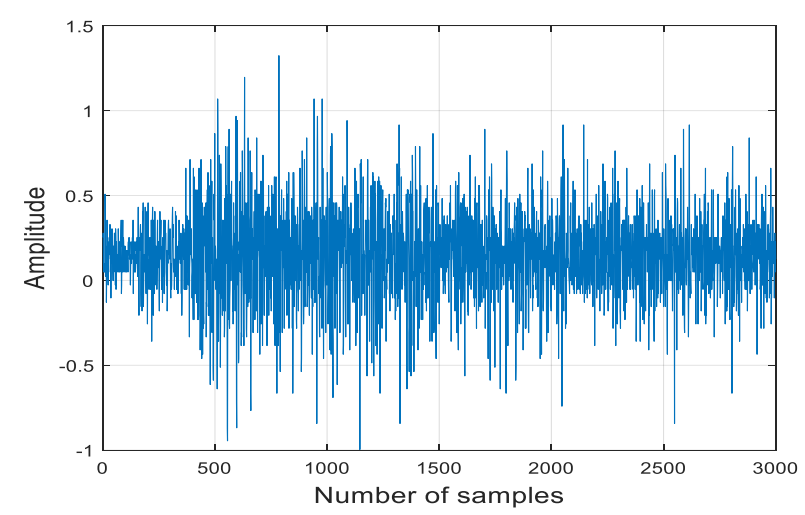

Fig. 3. Sample of EMG Signal.

Enhanced Mean Absolute Value (EMAV)

EMAV $=\frac{1}{L} \sum_{i=1}^{L}\left|\left(x_{i}\right)^{p}\right|$

$p=\left\{\begin{array}{l}0.75, \text { if } i \geq 0.2 L \& i \leq 0.8 L \\ 0.50, \text { otherwise }\end{array}\right.$

Enhanced Wavelength (EWL)

$\mathrm{EWL}=\sum_{i=2}^{L}\left|\left(x_{i}-x_{i-1}\right)^{p}\right|$
$p=\left\{\begin{array}{l}0.75, \text { if } i \geq 0.2 L \& i \leq 0.8 L \\ 0.50, \text { otherwise }\end{array}\right.$

where $x$ is the wavelet coefficient and $L$ is the length of the coefficient. As can be seen in Eq. (17) and (18), the parameter $p$ is used to identify the influence of sample within the signal. In EMAV and EWL, a greater number of $p$ is utilized for $20 \%$ to $80 \%$ of regions. This is because by strengthening the information content at the middle region, more valuable information can be obtained. In this way, the quality of features can be improved. Furthermore, it is seen that EMAV and EWL are the extension of MAV and WL with simple modification, and thus no much additional computational time is required in the evaluation.

\section{E. Machine Learning Algorithm}

After feature extraction, the EMG feature set is built. Four popular machine learning algorithms include $k$-nearest neighbor (KNN), Naïve Bayes (NB), linear discriminate analysis (LDA) and support vector machine (SVM) are employed to evaluate the effectiveness of proposed features in EMG pattern recognition. These machine learning algorithms are chosen due to their promising performances in previous works.

$\mathrm{KNN}$ is one of the famous learning algorithms, which performs faster than other modern algorithms in classification tasks [27]. Briefly, KNN is categorized into learning and classification phases. The former gathers the training data for training work, whereas the latter predicts the test data with all the training data according to the $k$ most similar training data [23]. In this work, the $k$-value of KNN is set at 1 . 
NB is a simple machine learning algorithm, and it is good at measuring the density of the dataset. Generally, NB employs the Bayes theorem to determine the probability of data by assuming all the features are independent [28]. It predicts the most probable class by examining the probability of test features. In this paper, NB with Gaussian kernel distribution is utilized.

LDA is the most robustness learning algorithm in EMG studies. Additionally, LDA is high speed training and computationally less expensive [29]. In LDA, the data is assumed to be normally distributed with equal covariance matrices. The main idea of LDA is to discriminate the observed features to the target class in which the posteriori probability can be maximized [25]. In this study, the LDA with pseudolinear function is utilized.

SVM is a well-known and powerful learning algorithm in EMG pattern recognition. The general idea of SVM is to seek out the hyperplane that partitions the data into desired class in which the data is transformed into high dimensional space [30]. However, SVM is designed for binary class, but not the multiclass problems. Therefore, the SVM with one-versus-all approach and radial basis kernel (RBF) function is applied in this paper [31].

\section{RESULTS AND DISCUSSIONS}

Remark, the features are extracted from the EMG signals via DWT transformation. For each feature type, 16 features (1 feature $\times 2$ channels $\times 8$ coefficients) are extracted from each movement from each subject. Afterward, the extracted features are fed into the KNN, NB, LDA and SVM for the classification process. In this work, 10 -folds cross-validation method is applied. In 10-folds cross-validation manner, the data is equally divided into 10 folds, where each fold is used for testing in succession, and the remainder 9 folds are used to train the classifier. Finally, the mean accuracy obtained from 10 folds is recorded.

In the first part of the experiment, the performance of single feature (EWL and EMAV) is examined, and the result is compared with other 14 conventional EMG features. Table II outlines the average accuracy of 16 features over five subjects. Note that the best result for each classifier is bolded. From Table II, the average accuracy achieved by the proposed features (EMAV and EWL) were much better than other conventional features for all learning algorithms. From the result obtained, it can be inferred that EMAV and EWL are powerful features in EMG signals classification.

Among the KNN, NB, LDA and SVM, the optimal learning algorithm is found to be LDA, which contributed the highest classification accuracy in this work. Inspecting the result on LDA, it is seen that EMAV and EWL contributed competitive performance in current work. Consequently, EWL and EMAV scored high average accuracy of $95.11 \%$ and 96.89\%. The result obtained indicates that EMAV and EWL features were able to provide promising performance in this research.

In the second part of the experiment, the efficacy of the combination of EWL and EMAV with other features is investigated. Table III demonstrates the average accuracy of 14 different feature combinations over five subjects. In this table, the best result for each classifier is highlighted with bold text. As can be observe, instead of using a single feature, the combination of EWL and EMAV with other features can effectively improve the prediction accuracy. By applying LDA, it is seen that the combination of EWL+EMAV+MAV+WL contributed the optimal average accuracy in differentiating the six different hand movements. Moreover, it is observed that EMAV+EWL+ZC+SSC achieved the best average accuracy when KNN, NB and SVM are utilized. On the whole, it can be inferred that the combinations of EMAV and EWL with other features were beneficial in improving the classification performance of EMG pattern recognition system.

Based on the result obtained, the best average accuracy is achieved by using the proposed combined EMG feature set (EWL+EMAV+MAV+WL) with LDA classifier, 97.56\%. In comparison with other conventional feature sets, the proposed feature set is highly capable in discriminating the EMG patterns, which leads to better classification result. On the other hand, the experimental results show the superiority of LDA against KNN, NB and SVM. This might because the extracted features consist of high linearity, thus resulting in high prediction accuracy.

TABLE II. AVERAGE ACCURACY OF 16 FEATURES OVER 5 SUBJECTS

\begin{tabular}{|l|l|l|l|l|}
\hline \multirow{2}{*}{ Feature } & \multicolumn{4}{|l|}{ Average accuracy $(\%)$} \\
\cline { 2 - 5 } & $K N N$ & $L D A$ & $S V M$ & $N B$ \\
\hline MAV & 94.56 & 96.33 & 95.11 & 93.22 \\
\hline WL & 94.56 & 94.56 & 95.89 & 93.78 \\
\hline ZC & 94.22 & 95.11 & 95.00 & 92.56 \\
\hline SSC & 83.89 & 87.22 & 89.00 & 83.67 \\
\hline AAC & 92.56 & 94.56 & 94.44 & 93.78 \\
\hline LD & 90.22 & 91.78 & 92.78 & 91.56 \\
\hline RMS & 94.00 & 95.67 & 95.11 & 93.78 \\
\hline DASDV & 93.56 & 95.89 & 95.11 & 93.22 \\
\hline MYOP & 81.00 & 86.33 & 86.89 & 84.33 \\
\hline WA & 75.67 & 81.56 & 79.67 & 80.78 \\
\hline SSI & 93.00 & 90.00 & 94.78 & 92.44 \\
\hline VAR & 90.56 & 90.00 & 92.44 & 92.44 \\
\hline MMAV & 94.33 & 95.44 & 95.44 & 93.00 \\
\hline MMAV2 & 91.44 & 93.56 & 93.56 & 92.11 \\
\hline EWL & $\mathbf{9 5 . 0 0}$ & 95.11 & $\mathbf{9 6 . 2 2}$ & 93.56 \\
\hline EMAV & 94.67 & $\mathbf{9 6 . 8 9}$ & 96.11 & $\mathbf{9 3 . 8 9}$ \\
\hline
\end{tabular}

TABLE III. AVERAGE ACCURACY OF 14 FEATURE COMBINATIONS OVER 5 SUBJECTS

\begin{tabular}{|l|l|l|l|l|}
\hline \multirow{2}{*}{ Feature combination } & \multicolumn{4}{|l|}{ Average accuracy $\mathbf{\%})$} \\
\cline { 2 - 5 } & $K N N$ & $L D A$ & $S V M$ & $N B$ \\
\hline EMAV+EWL & 95.00 & 96.89 & 96.22 & 94.56 \\
\hline EMAV+EWL+MAV+WL & 94.44 & $\mathbf{9 7 . 5 6}$ & 96.22 & 94.22 \\
\hline EMAV+EWL+SSC+ZC & $\mathbf{9 6 . 0 0}$ & 95.22 & $\mathbf{9 7 . 2 2}$ & $\mathbf{9 6 . 5 6}$ \\
\hline EMAV+EWL+MYOP+WA & 95.22 & 95.11 & 96.33 & 93.89 \\
\hline EMAV+EWL+RMS+DASDV & 95.00 & 96.67 & 96.22 & 94.78 \\
\hline WL+MAV+SSC+ZC & 95.33 & 96.11 & 96.78 & 96.44 \\
\hline MYOP+WA+SSI & 93.00 & 89.78 & 94.67 & 93.67 \\
\hline MAV+WL+MYOP+WA & 94.67 & 95.33 & 96.11 & 93.67 \\
\hline MAV+MMAV+MMAV2 & 94.22 & 94.56 & 95.67 & 93.67 \\
\hline AAC+LD+RMS+DASDV & 94.22 & 96.11 & 95.22 & 94.00 \\
\hline WL+MAV & 94.56 & 96.33 & 95.89 & 94.11 \\
\hline AAC+LD+MYOP+WA & 77.00 & 94.22 & 81.00 & 92.78 \\
\hline SSI+VAR+MMAV+MMAV2 & 93.00 & 95.44 & 94.67 & 93.56 \\
\hline MYOP+WA & 75.78 & 85.78 & 79.67 & 86.67 \\
\hline
\end{tabular}




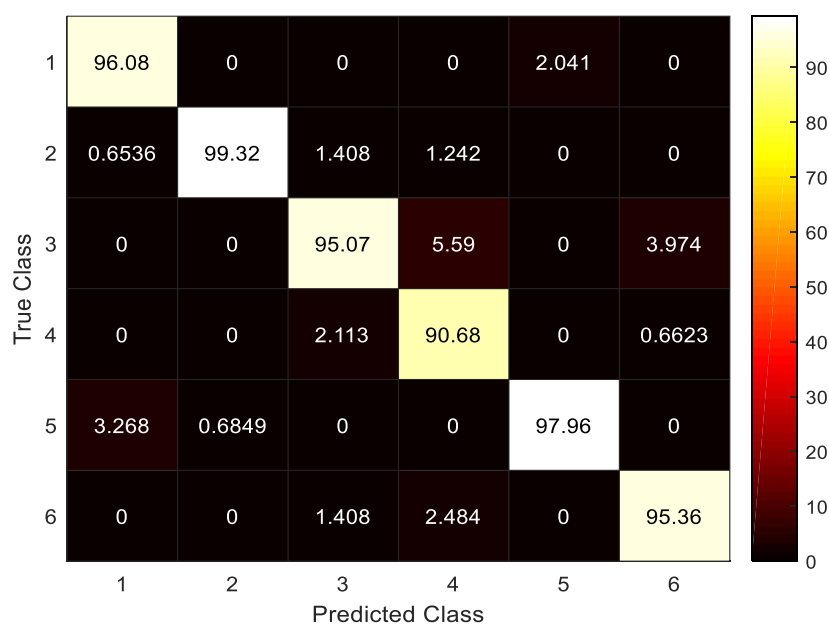

Fig. 4. Confusion Matrix of Combined Feature Set (EMAV+EWL+MAV+WL) with LDA over 5 Subjects (\%).

Furthermore, the result of class-wise accuracy (accuracy of each hand movement task) is discussed. Fig. 4 illustrates the confusion matrix of proposed combined feature set (EMAV+EWL+MAV+WL) with LDA over five subjects. Note that the number 1 to 6 in both axes represents the hand movement types. From Fig. 4, it is observed that the tip movement $\left(2^{\text {nd }}\right.$ hand movement task) has been perfectly recognized $(99.32 \%)$, followed by cylindrical movement $(97.96 \%)$. The results obtained evidently show the efficacy of EWL and EMAV in EMG signals classification. Hence, EWL and EMAV features can be appropriate choices for EMG feature extraction in clinical and rehabilitation applications.

\section{CONCLUSION}

The quality of feature is one of the important factors that can greatly affect the accuracy in EMG signals classification. In this paper, two modified versions of WL and MAV features, namely EWL and EMAV are proposed for efficient EMG signals classification. The proposed features are tested with the EMG data of five healthy subjects, and the results are further compared with other conventional EMG features. In addition, the performance of the combinations of EWL and EMAV with other features are also investigated. Our results showed that the best classification performance was achieved by the LDA and combined feature set (EWL+EMAV+MAV+WL). In short, EWL and EMAV can be the valuable tools in EMG pattern recognition.

There are several limitations in this work. First, only five combinations of feature sets (with EMAV and EWL) are applied. However, it is worth noting that the combination of EMAV and EWAL with other EMG features can be also considered for performance enhancement. Second, the scope of this study is limited to one time-frequency method, DWT. However, other popular time-frequency methods such as wavelet packet transform (WPT) and empirical mode decomposition (EMD) are also applicable to EMG signal processing. In the future, the EWL and EMAV can be extracted directly from EMG signals without performing the transformation. Therefore, more effective EMG pattern recognition system can be developed in future work.

\section{ACKNOWLEDGMENT}

The authors would like to thank the Ministry of Higher Education (MOHE), Malaysia for funding research under grant GLuar/STEVIA/2016/FKE-CeRIA/100009.

\section{REFERENCES}

[1] Y. Gu, D. Yang, Q. Huang, W. Yang, and H. Liu, "Robust EMG pattern recognition in the presence of confounding factors: features, classifiers and adaptive learning," Expert Syst. Appl., vol. 96, pp. 208-217, Apr. 2018.

[2] M. F. Wahid, R. Tafreshi, M. Al-Sowaidi, and R. Langari, "Subjectindependent hand gesture recognition using normalization and machine learning algorithms," J. Comput. Sci., vol. 27, pp. 69-76, Jul. 2018.

[3] N. Wang, K. Lao, X. Zhang, J. Lin, and X. Zhang, "The recognition of grasping force using LDA," Biomed. Signal Process. Control, vol. 47, pp. 393-400, Jan. 2019.

[4] G. Gaudet, M. Raison, and S. Achiche, "Classification of Upper limb phantom movements in transhumeral amputees using electromyographic and kinematic features," Eng. Appl. Artif. Intell., vol. 68, pp. 153-164, Feb. 2018.

[5] Y. Ning and Y. Zhang, "A new approach for multi-channel surface EMG signal simulation," Biomed. Eng. Lett., vol. 7, no. 1, pp. 45-53, Feb. 2017.

[6] B. Hudgins, P. Parker, and R. N. Scott, "A new strategy for multifunction myoelectric control.," IEEE Trans. Biomed. Eng., vol. 40, no. 1, pp. 82-94, Jan. 1993.

[7] R. N. Khushaba, M. Takruri, J. V. Miro, and S. Kodagoda, "Towards limb position invariant myoelectric pattern recognition using timedependent spectral features," Neural Netw., vol. 55, no. Supplement C, pp. 42-58, Jul. 2014.

[8] O. W. Samuel et al., "Pattern recognition of electromyography signals based on novel time domain features for amputees' limb motion classification," Comput. Electr. Eng., vol. 67, pp. 646-655, Apr. 2018.

[9] K. Xing, P. Yang, J. Huang, Y. Wang, and Q. Zhu, "A real-time EMG pattern recognition method for virtual myoelectric hand control," Neurocomputing, vol. 136, no. Supplement C, pp. 345-355, Jul. 2014.

[10] K. Englehart, B. Hudgins, P. A. Parker, and M. Stevenson, "Classification of the myoelectric signal using time-frequency based representations” Med. Eng. Phys., vol. 21, no. 6, pp. 431-438, Jul. 1999.

[11] A. Subasi, "Classification of EMG signals using combined features and soft computing techniques," Appl. Soft Comput., vol. 12, no. 8, pp. 2188-2198, Aug. 2012.

[12] J. Too, A. R. Abdullah, N. Mohd Saad, and N. Mohd Ali, "Feature Selection Based on Binary Tree Growth Algorithm for the Classification of Myoelectric Signals," Machines, vol. 6, no. 4, p. 65, Dec. 2018.

[13] J. Too, A. R. Abdullah, N. M. Saad, N. M. Ali, and T. N. S. T. Zawawi, "Exploring the Relation Between EMG Pattern Recognition and Sampling Rate Using Spectrogram,” J. Electr. Eng. Technol., vol. 14, no. 2, pp. 947-953, Mar. 2019.

[14] M. R. Canal, "Comparison of Wavelet and Short Time Fourier Transform Methods in the Analysis of EMG Signals," J. Med. Syst., vol. 34, no. 1, pp. 91-94, Feb. 2010.

[15] A. Phinyomark, C. Limsakul, and P. Phukpattaranont, "Application of Wavelet Analysis in EMG Feature Extraction for Pattern Classification," Meas. Sci. Rev., vol. 11, no. 2, pp. 45-52, 2011.

[16] A. B. M. S. U. Doulah, S. A. Fattah, W. P. Zhu, and M. O. Ahmad, "Wavelet Domain Feature Extraction Scheme Based on Dominant Motor Unit Action Potential of EMG Signal for Neuromuscular Disease Classification," IEEE Trans. Biomed. Circuits Syst., vol. 8, no. 2, pp. 155-164, Apr. 2014.

[17] "UCI Machine Learning Repository." Website: https://archive.ics.uci.edu/ml/index.php.

[18] C. Sapsanis, G. Georgoulas, A. Tzes, and D. Lymberopoulos, "Improving EMG based classification of basic hand movements using EMD," in 2013 35th Annual International Conference of the IEEE Engineering in Medicine and Biology Society (EMBC), 2013, pp. 57545757. 
[19] A. Subasi, "Classification of EMG signals using PSO optimized SVM for diagnosis of neuromuscular disorders," Comput. Biol. Med., vol. 43, no. 5, pp. 576-586, Jun. 2013.

[20] E. Gokgoz and A. Subasi, "Comparison of decision tree algorithms for EMG signal classification using DWT," Biomed. Signal Process. Control, vol. 18, pp. 138-144, Apr. 2015.

[21] J. Too, A. R. Abdullah, and N. M. Saad, "A Comparative Analysis of Wavelet Families for the Classification of Finger Motions," Int. J. Adv. Comput. Sci. Appl. IJACSA, vol. 10, no. 4, 2019.

[22] A. Phinyomark, F. Quaine, S. Charbonnier, C. Serviere, F. TarpinBernard, and Y. Laurillau, "EMG feature evaluation for improving myoelectric pattern recognition robustness," Expert Syst. Appl., vol. 40, no. 12, pp. 4832-4840, Sep. 2013.

[23] W.-T. Shi, Z.-J. Lyu, S.-T. Tang, T.-L. Chia, and C.-Y. Yang, "A bionic hand controlled by hand gesture recognition based on surface EMG signals: A preliminary study," Biocybern. Biomed. Eng., vol. 38, no. 1, pp. 126-135, Jan. 2018.

[24] A. Phinyomark, P. Phukpattaranont, and C. Limsakul, "Feature reduction and selection for EMG signal classification," Expert Syst. Appl., vol. 39, no. 8, pp. 7420-7431, Jun. 2012.

[25] D. Tkach, H. Huang, and T. A. Kuiken, "Study of stability of timedomain features for electromyographic pattern recognition," J. NeuroEngineering Rehabil., vol. 7, p. 21, May 2010.
[26] K. S. Kim, H. H. Choi, C. S. Moon, and C. W. Mun, "Comparison of knearest neighbor, quadratic discriminant and linear discriminant analysis in classification of electromyogram signals based on the wrist-motion directions," Curr. Appl. Phys., vol. 11, no. 3, pp. 740-745, May 2011.

[27] L.-Y. Chuang, C.-H. Yang, and J.-C. Li, "Chaotic maps based on binary particle swarm optimization for feature selection," Appl. Soft Comput., vol. 11, no. 1, pp. 239-248, Jan. 2011.

[28] P. A. Karthick, D. M. Ghosh, and S. Ramakrishnan, "Surface electromyography based muscle fatigue detection using high-resolution time-frequency methods and machine learning algorithms," Comput. Methods Programs Biomed., vol. 154, no. Supplement C, pp. 45-56, Feb. 2018.

[29] D. Rivela, A. Scannella, E. E. Pavan, C. A. Frigo, P. Belluco, and G. Gini, "Analysis and Comparison of Features and Algorithms to Classify Shoulder Movements From sEMG Signals," IEEE Sens. J., vol. 18, no. 9, pp. 3714-3721, May 2018.

[30] A.-C. Tsai, J.-J. Luh, and T.-T. Lin, “A novel STFT-ranking feature of multi-channel EMG for motion pattern recognition," Expert Syst. Appl., vol. 42, no. 7, pp. 3327-3341, May 2015.

[31] T. Lorrain, N. Jiang, and D. Farina, "Influence of the training set on the accuracy of surface EMG classification in dynamic contractions for the control of multifunction prostheses," J. NeuroEngineering Rehabil., vol. 8, p. 25, May 2011. 